\title{
Dynamic stabilization devices in the treatment of low back pain
}

\author{
D. K. Sengupta \\ Dartmouth-Hitchcock Medical Centre, One Medical Centre Drive, Lebanon, NH, USA
}

Soft stabilization has an important role in the treatment of the degenerative lumbar spine. Fusion of one or two motion segments may not make a big difference in the total range of motion of the lumbar spine, but preserving flexibility of a motion segment may prevent adjacent segment disease and may permit disc replacement, even when facet joints need to be excised. A favourable environment is created in the motion segment by unloading the disc and permitting near normal motion, the disc may be able to repair itself or may supplement reparative potential of gene therapy.

Key words: Vertebrae, intervertebral disk displacement, low back pain

\section{Introduction}

The conventional surgical treatment for chronic low back pain due to degenerative disorders in the lumbar spine is spinal fusion. This may be compared to arthrodesis of the hip or knee for severe osteoarthritis of those joints, as practiced in the past. Arthrodesis of the hip and knee leads to major disability of locomotor function and gait, and has long since been discarded. Spinal fusion has been practiced for several decades for similar indications, since overall movement of the trunk, as well as gait, is only minimally affected after fusion of one or two segments in the lumbar spine.

With recent developments of the fusion techniques, successful fusion rate has approached nearly $100 \%$ but this has failed to reflect a comparable increase in the successful clinical outcome. ${ }^{[1]}$ Several studies in the recent literature questioned the efficacy of spinal fusion in the treatment of low back pain. ${ }^{[2]}$ In addition, a significant apprehension of adjacent segment disease in the long term follow-up after spinal fusion always concerned the spinal surgeon. ${ }^{[3-8]}$

Stabilization of the lumbar spine without fusion has been sporadically practiced during the last decade in the Europe and the East ${ }^{[9-10]}$ (or West). The demand for an ideal dynamic stabilization system has increased for younger patients with multisegment disc degeneration, where adjacent segment disease may be more likely to happen following fusion in the long-term follow-up. The interest in dynamic stabilization has grown the development of indications like topping off an adjacent segment to or stabilizing a segment following discectomy. Unfortunately, neither of these procedures has a solid biomechanical basis for action, nor there has been any clinically proven efficacy as yet. Their popularity is based more on lack of satisfaction with conventional spinal fusion rather than proven superiority.

The prosthetic replacement of the disc has made its way in the therapeutic armamentarium of degenerative disc disease following the natural course of evolution with the success of hip and knee replacement. It is more than a decade that several designs of disc prostheses have been used clinically, some of which have over five years of clinical follow-up. The overall clinical success rate, however, is no match to that of hip or knee arthroplasty, and even struggles to match the results of conventional spinal fusion.

The basic difference between prosthetic replacement of the hip or knee and the intervertebral disc prosthesis is that the latter is only a partial rather than a total joint replacement. The disc prosthesis has to share the kinematics of the remaining.joints of the spinal motion segment, very much like the other dynamic stabilization devices. The primary difference is that the disc prosthesis is a load bearing structure as opposed to the load sharing nature of the dynamic stabilization devices. Based on the experience with the hip and knee arthroplasty, where the wear property of the plastic component is closely related to the osteolysis, much attention has been paid to the wear properties of the available disc prosthesis. However, the kinematies' effect of the disc prosthesis on the remaining structures of the motion segment has been largely ignored.

Pathology and mechanism of chronic low back pain - movement or load. 


\section{Mechanical back pain}

Traditionally chronic axial pain in the low back due to degenerative diseases of the lumbar spine, which is aggravated by daily activities, is described as 'mechanical back pain'. This is often thought to be due to instability of the lumbar motion segment, secondary to the dise degeneration and facet arthrosis. In contrast, symptoms of leg pain, which is described as either radicular pain or neurogenic claudication, have been ascribed to compression of the neural elements.

\section{Spinal instability}

Instability in the lumbar spine as a cause of mechanical back pain is poorly understood. The term instability would imply an abnormal motion under physiological load. It is generally accepted that disc degeneration reduces the movement of the lumbar spine, except in the very early stages. ${ }^{[11-12]}$ Despite considerable efforts over many years, no clear relationship has been established between low back pain and abnormal movement. ${ }^{[13-18]}$ Most authors related abnormal translation to mechanical back pain. ${ }^{[11-12,17-18]}$ A degree of translation motion is normally associated with flexion-extension due to the composite nature of spinal motion. ${ }^{[19-20]}$ While some investigators found excessive translation to be associated with dise degeneration, ${ }^{[18]}$ others observed a reduced shear-flexion ratio at the symptomatic levels of dise degeneration. ${ }^{[17]}$ It is generally accepted that the effect of disc degeneration is to reduce the movement, not to increase it, as the term "instability" would imply. ${ }^{[11-12,21]}$

While abnormal translation is seen radiologically in many cases of dise degeneration with spondylolisthesis, it is not always present in symptomatic dise degeneration. Even when it is present, pain may not be continuous. In other words, although the abnormal movement may be present continuously in this group of patients, their pain is not continuous. It is therefore difficult to find any basis for the concept of abnormal movement or instability as a cause of back pain. 'Instability' was a phrase that clinicians used since the last century to imply that there was some non-specific mechanical failure of the spine causing back pain, as opposed to infection or arthritis. Bioengineers define instability in a purely literal sense, with abnormal movement at the joint surface and that indicated fusion as an appropriate solution. ${ }^{[21]}$

\section{Load transmission through the normal disc}

The second important role of a motion segment in the lumbar spine is load transmission. Under normal circumstances load transmission is painless. The magnitude of load transmission through the lumbar motion segment varies with change of posture and activities. This has been very well documented by the earlier work of Nachemson. ${ }^{[22]}$ A recent in vivo intradiscal pressure study in a volunteer by Wilke et al. ${ }^{[23]}$, in different posture and activities, found a good correlation with Nachemson' ${ }^{[22]}$ data.

Normal intervertebral dise is an isotropic structure. ${ }^{[24]}$ The nucleus acts almost like a fluid filled bag and distributes load uniformly across the endplate. This has been convineingly demonstrated by McMillan et al. ${ }^{[25]}$ and McNally and Adams ${ }^{[26]}$ by stress profilometry in normal cadaver lumbar dise (Figures 1 , 2 ). The nucleus also transmits the load in the lateral direction to the annulus producing tension in the annulus, similar to an inflated car tire. This converts the annulus into a uniform load bearing structure. ${ }^{[21]}$

Disc degeneration alters the isotropic nature of the disc. ${ }^{[28]} I n$ vivo stress profilometry studies also confirm that load distribution across the endplate becomes uneven after disc degeneration, with areas of high spot loading, particularly associated with certain positions (Figure 2C). Such irregular load distribution was found to be a strong predictor of pain reproduction by provocative discography ${ }^{[27]}$.

The irregular load distribution across the joint surface is a wellrecognized cause of pain in diarthrodial joints like hip and knee joint. High spot loading in varus or valgus deformity of the knee joint may lead to pain, and this may be relieved by corrective osteotomy, which redistributes the load transmission. Similarly, high spot loading in a subluxating hip may cause pain, which may be relieved by osteotomy and redistribution of load transmission. Although the intervertebral disc is not a diarthrodial joint, the endplates are rich in nerve supply, and it may be reasonable to believe that abnormal high spot loading may be a cause of pain in degenerative disc disease.

\section{Load transmission through the degenerated disc}

The normal nucleus consists of a homogeneous gel of collagen and proteoglycan. In a degenerated disc, it changes to a nonhomogeneous mixture of fragmented and condensed collagen, areas of fluid, and indeed areas of gas on occasion. Less commonly, isolated fragments of annulus or endplate may add to the loose fragment inside the disc. ${ }^{[29]}$ In a degenerated disc, the nucleus becomes depressurized and an increasingly larger load is transmitted through the annulus. Corresponding endplate changes, apoptosis of cells, ${ }^{[30]}$ destruction and thinning of the trabeculae in the central areas, ${ }^{[31]}$ and thinning of the subchondral cartilaginous endplate ${ }^{[32]}$ have been demonstrated by various observers. Loading the annulus, unprotected by the supporting pressure of the nucleus, leads to splitting and inward folding of

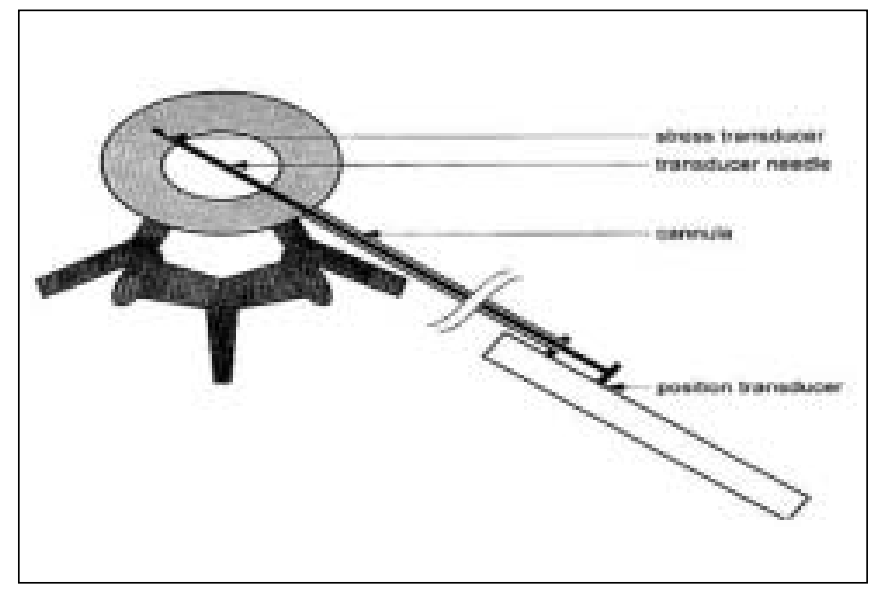

Figure 1: Schematic diagram of the equipment and approach used in the in vivo stress measurements ${ }^{[27]}$ 

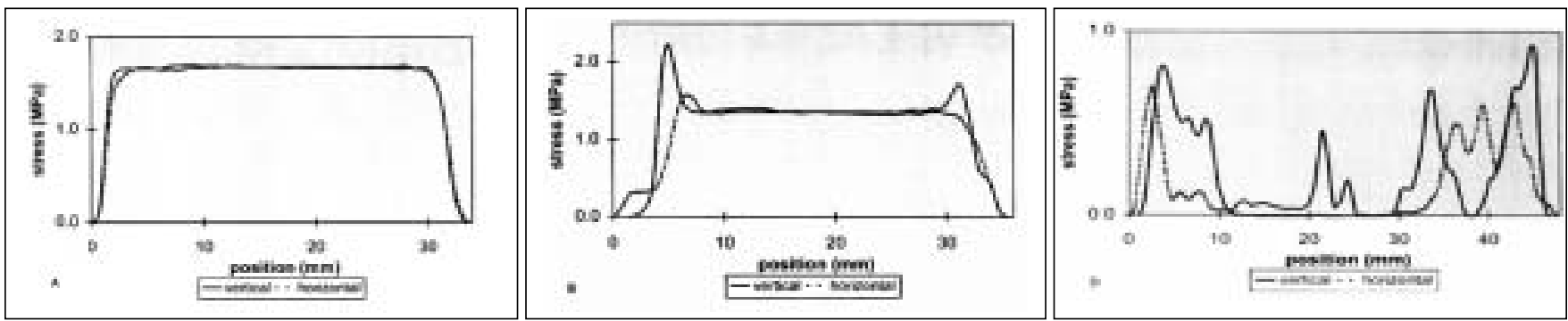

Figure 2: A. A pair of stress profiles recorded in a healthy L4-L5 disc in vitro showing a uniform, isotropic distribution of compressive stress within the nucleus and a rapid fall off in the peripheral annulus. B. A pair of stress profiles recorded in a degenerate L2-L3 disc in vitro showing anisotropic concentrations of stress in the annulus. C. A pair of stress profiles recorded in an L5-S1 disc in vivo showing multiple stress concentrations in the posterolateral annulus and in the otherwise depressurized nucleus ${ }^{[27]}$

the annulus. ${ }^{[33]}$ Areas of main load transmission across the disc now become dependent on the posture; in flexion the anterior annulus, in extension the posterior annulus. The abnormal high load transmission through the different areas of the annulus with change of posture may explain the activity and posture related mechanical back pain in the presence of degenerated disc.

\section{'Stone in the shoe' hypothesis of back pain}

In the clinical scenario of mechanical back pain two commonly observed facts remains unexplained by the concept of instability. First, patients with disc degeneration almost always have a mild to moderate degree of baseline symptom, superimposed with periods of acute exacerbations of back pain. Patients are relatively asymptomatic between the acute episodes. If there is any abnormal translation or instability in that segment, it is present continuously. It is difficult to understand if instability or abnormal movement is the cause of pain, therefore then is the acute pain is only periodic. Secondly, it is a well-recognized fact that manipulation of the lumbar spine, as practiced by chiropractors, often results in dramatic relief of an acute episode of low back pain. The instability concept again fails to explain such observation. ${ }^{[21]}$

As described earlier, in a degenerated disc, the nucleus looses its water content, becomes depressurized, and the opposing endplate cartilage and the supporting trabeculae thins out. However, the fragments of nucleus or endplate cartilage, or annulus in a degenerated disc may, on occasion, become areas of high spot loading, depending on their position within the disc. It is tempting to hypothesize that these abnormal loose fragments within the disc space act like a stone in the shoe, causing high spot loading and pain at the time of acute exacerbation. Manipulation of the lumbar spine by a chiropractor may, on occasion, dislodge the fragment from its weight-bearing position, bringing immediate relief of acute pain. ${ }^{[21]}$

The above hypothesis, that the actual arrangement of tissues within the disc affects the load transmission, also explains the fact that there is no clear correlation between degree of disc degeneration and pain.

\section{Spinal fusion and low back pain}

It is true that spinal fusion in many cases successfully relieve back pain. Following spinal fusion, the load transmission across the motion segment becomes direct from bone to bone. However, if instability or an abnormal movement would be the main cause of back pain, one would expect that spinal fusion would always be successful in relieving back pain. Unfortunately, this has not been the clinical experience. Introduction of pedicle instrumentation in the $1980 \mathrm{~s}$, and cage devices in the $1990 \mathrm{~s}$, and frequent use of circumferential fusion have increased the successful fusion rate close to $98 \%$, but failed to improve the overall clinical success rate ${ }^{[1]}$ A recent review of the Cochrane database of the prospective randomized controlled trials fails to establish any significant improvement of the natural history of low back pain by spinal fusion. ${ }^{[2]}$ A subsequent multicentric prospective randomized controlled trial in Sweden suggests better outcome with fusion compared to conservative treatment, but no difference in outcome between the different fusion techniques. ${ }^{[34]}$

The debate continues and fusion remains the seriously challenged standard surgical treatment of back pain. Most reported studies suggest 50-70\% excellent to good clinical outcome, and $30 \%$ failure of improvement of back pain, ${ }^{[35-43]}$ but no correlation between fusion success and clinical outcome. Neither are all cases of pseudarthrosis painful, nor all successful fusion painless.

Many suggestions have been put forward to explain failure of good clinical outcome in the presence of a successful fusion. Those include failure to recognize pseudarthrosis, ${ }^{[44]}$ abnormal sagittal balance, ${ }^{[45]}$ and abnormal load transmission through the metalbone interface in cage fusion. ${ }^{[46]}$ The load over the narrow footprint of the metallic cage on the endplate may be $500 \%$ higher than the rest of the endplate, even after successful fusion. ${ }^{[46]} \mathrm{McAfee}{ }^{[44]}$ pointed out that clinical success was associated with the development of the bone around the cage, inevitably increasing the area of load transmission and reducing the load over the footprint of the cage.

It may therefore be reasonable to conclude that stopping movement is not the factor in achieving relief of back pain. Creating a normal loading pattern is more important for clinical success. This leads to the era of a new look into the problem of mechanical low back pain, which is 'stabilize but do not fuse'.

\section{Dynamic stabilization Definition}

Dynamic stabilization of the lumbar spine may be defined as a system, which would favourably alter the movement and load transmission of a spinal motion segment, without the intention of 
fusion of the segment.

This needs to be carefully differentiated from semi rigid fixation of the spine, where a fusion is intended. It is often argued that a rigid fixation may discourage formation of a strong fusion mass due to load sharing by the rigid implant, and a semi rigid instrumentation may overcome this disadvantage. A dynamic stabilization, also known as soft stabilization or flexible stabilization, leaves the spinal segment mobile, and its intention is to alter the load bearing pattern of the motion segment, as well as to control any abnormal motion at the segment.

\section{The principles of dynamic stabilization}

The hypothesis behind dynamic stabilization is that control of abnormal motions and more physiological load transmission would relieve pain, and prevent adjacent segment degeneration. A remote expectation is that, once normal motion and load transmission is achieved, the damaged disc may repair itself, unless of course the degeneration is too advanced.

The pertinent questions in dynamic stabilization therefore are a) how much control of motion is desirable, and b) how much load should be shared by the system, to unload the damaged disc. The question in the long term is, how to prevent implant failure, in view of constant movement of the stabilized segment. A pseudarthrosis often leads to fatigue failure of implants used for rigid fixation, because the rigidity of the implant does not permit them to accommodate any motion originated at the pseudarthrosis. Flexible stabilization may accommodate this movement, and may avoid a fatigue failure. However, a closer look at the kinematics of the dynamic stabilization leads to further consideration before its fatigue life may be determined.

The dynamic stabilization system has to permit motion and also share load. The load sharing by should be more or less uniform during the entire range of motion. This may be achieved when the location of the optimum instant axis of rotation (IAR) of the system lies close to that of the motion segment during the entire range of motion. Any discrepancy in the kinematics between the dynamic stabilization system and the motion segment would lead to variability of load-sharing property.

Normally the disc is loaded both during flexion and extension. The average disc pressure rises during flexion and also during extension, and is lowest during the early phase of extension. ${ }^{[23]}$ Let us consider a dynamic stabilization system that shares $30 \%$ of the load during flexion, allowing only $70 \%$ of the load to pass through the damaged disc; during extension, if the system forces the IAR to be shifted posterior to the disc, the disc will be distracted more and more towards the end of extension. This will be evident by a progressive lowering of the disc pressure towards the end of extension, which indicates that the dynamic stabilization system becomes an increasingly load-bearing structure in extension (Figure 3). This may lead to fatigue failure of the implant or the implant-bone junction. ${ }^{[47]}$ It has been discussed later that the relatively rigid dynamic stabilization devices like DSS-I, dynesis and interspinous spacers may have this disadvantage.

Conversely, the ligament based dynamic stabilization systems

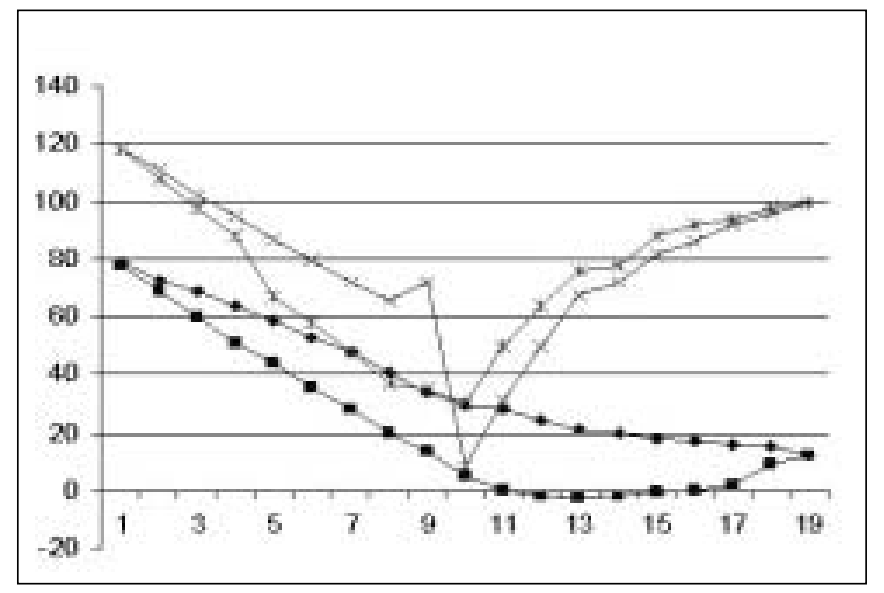

Figure 3: Continuous pressure measurement from the centre of the disc at L4-5 segment in a cadaveric lumbar spine during flexionextension motion. In unconstrained specimen, the pressure [the upper curve $(x)$ ] raises both in flexion and extension. Following application of a soft stabilisation system (dynamic stabilization system, DSS-I) the pressure in the disc [the lower curve (black dots)] was lowered during flexion proportionately, due to load-sharing property of the soft stabilization device. However, during extension the disc pressure continued to be lowered, except at the end of extension, indicating that the soft stabilization device became more and more load-bearing structure toward the end of extension

(e.g., graf ligament) may load-share during flexion, but they become lax during extension, and may not unload the disc at all during extension. Since these implants do not behave like a progressively increasing load-bearing structure, implant loosening is unlikely. This explains the fact that screw loosening has not been a problem with graf ligament stabilization ${ }^{[48]}$.

The disc prosthesis or nucleus prosthesis obeys the biomechanical principles of dynamic stabilization in uniform load transmission and preserving movement of a motion segment. But they differ by the fact that they replace a component of the motion segment, while the dynamic stabilization system does not.

\section{The dynamic stabilization devices}

The various dynamic stabilization systems described in the literature are all posterior implant. (Table 1) lists the various dynamic stabilization systems available.

The dynamic stabilization devices that have been described from time to time and used clinically may be classified into four categories (Table 1).

1. The Interspinous distraction devices are floating devices, i.e., not rigidly connected to the vertebrae. This avoids the possibility of loosening, a major concern for any implant which would have to survive against motion. They work by distraction of the spinous processes. Minns and Walsh ${ }^{[49]}$ described silicone interspinous spacers, which on biomechanical testing using cadaver in spine showed unloading of the disc and correction of the sagittal plane imbalance of the spine. No clinical application of this system has since been described by the authors.

Senegas et al. ${ }^{[50]}$ described a similar interspinous spacer in 1988, made of titanium, and held between the spinous processes with a dacron tape. After initial success in over three hundred patients, 


\section{Table 1: The different dynamic stabilization systems, that have been used clinically may be classified into four categories}

\section{Inter-spinous distraction devices}

Minns silicone distraction device

Wallis system

X-stop

Inter-spinous ligament devices

Elastic ligament (Bronsard's Ligament across the spinous processes)

Loop system

Ligaments across pedicle screws

Graf ligament

Dynesis device

Semi rigid metallic devices across the pedicle screws FASS system DSS system

the authors improved the system in the second generation, known as the 'Wallis implant' (Figure 4) which uses PEEK (polyetheretherketone) material as the spacer instead of titanium. The interspinous spacer blocks extension of the segment, and by distraction between the spinous processes, it holds the segment in relative flexion, a posture known to relieve neurogenic claudication pain in spinal stenosis. The Dacron tape acts to retain the plastic spacer in place, and limit further flexion of the segment. The device therefore more appropriately described as a hybrid of interspinous distraction device and interspinous ligament, described later. The clinical trials of the first-generation implant provided evidence that the interspinous system of dynamic stabilization is efficient against low-back pain due to degenerative instability and free of serious complications. A randomized clinical trial and an observational study of the new implant are currently underway by the same authors. The authors recommend the Wallis system for lumbar disc disease in the following indications: (i) discectomy for massive herniated disc leading to substantial loss

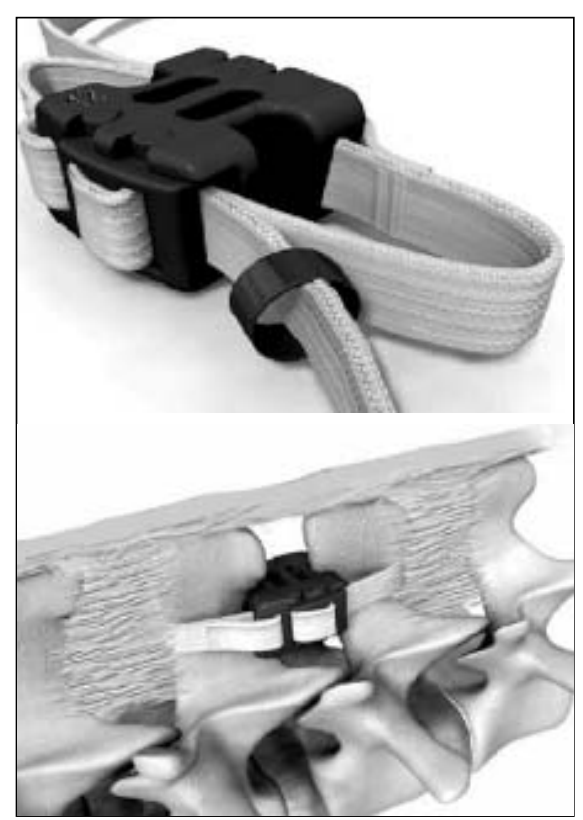

Figure 4: The Schematic view of the Wallis implant ${ }^{[50]}$ of disc material, (ii) a second discectomy for recurrence of herniated disc, (iii) discectomy for herniation of a transitional disc with sacralization of L5, (iv) degenerative disc disease at a level adjacent to a previous fusion, and (v) isolated Modic I lesion leading to chronic low-back pain.

Another titanium interspinous distraction device 'X-stop' (Figure 5) has been described by St. Francis Medical Technologies, Inc. ${ }^{[51]}$ which has completed FDA clinical trials, and is awaiting approval. However, no report of this device is available yet in the peer reviewed literature. The implant has been described for use in the spinal stenosis cases, where the interspinous distraction holds the spine into flexion, and thereby reduce the spinal stenosis. This device may be introduced by a minimally invasive approach, under local anesthetic, and may be useful for degenerative spinal stenosis in elderly patients with comorbid conditions, where it is preferable to avoid open surgery.

2 . The interspinous ligaments may be applied directly around the spinous processes, without using any metal anchorage. Caserta et al. ${ }^{[52]}$ reported their experience of using elastic ligament using alone or to supplement the adjacent segment to fusion (Figure 6). The authors have used the system in 82 cases since 1994 and described encouraging results. Unfortunately, their report does not describe any detail of the implant material or the clinical results.

Garner et al. ${ }^{[53]}$ described a new tension-band device for stabilization of the spine across the spinous processes, 'the loop system' (Spineology Inc., stillwater, MiNn.). It consists of a braided polyethylene cable, a locking clip, and an optional ferrule that can be placed in the spinous process. After tension is applied by use of a tensioning tool, a locking clip secures the construct (Figure 7). They compared static tensile and fatigue strength, stiffness, and creep properties, and compared the literature data for different spine cables and wire fixation systems. The authors concluded that 'the loop system' has strength similar to the titanium cable system and has a no-slip locking clip designed to maintain the construct tension. It combines the advantages of the metallic system

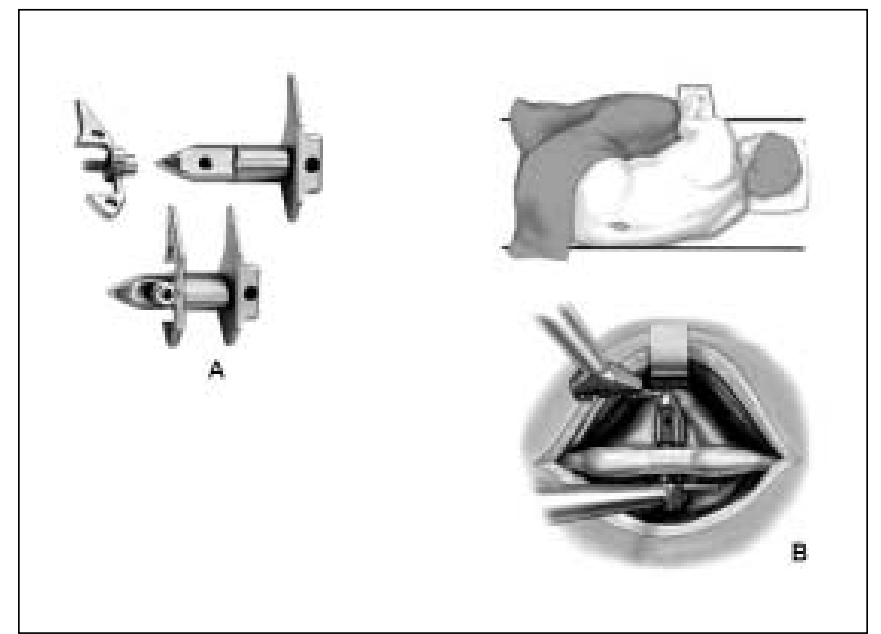

Figure 5: The 'X-stop' as introduced by the St. Francis Medical Technologies Inc. A. The implants, B. The schematic representation of surgical implantation ${ }^{[51]}$ 


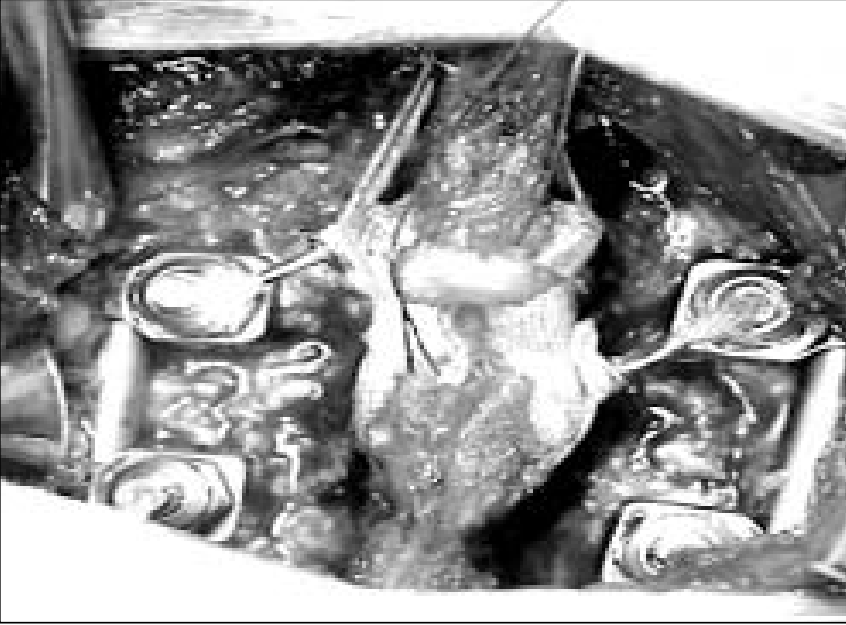

Figure 6: The elastic interspinous ligament as described by Caserta et al. ${ }^{[52]}$, peroperative picture of L4-5 elastic stabilization following rigid fixation of the L5-S1 segment

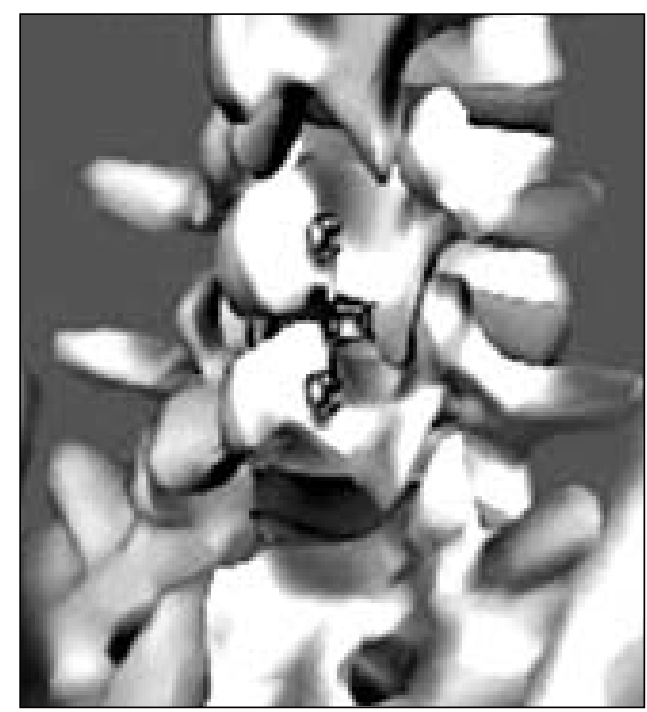

Figure 7: The loop system ${ }^{[2]}$

(lower creep) with the advantages of the polymer cables (high fatigue strength) without sacrificing strength, stiffness or ease of use.

3. Ligaments across the pedicles

4. Henry or Henri?

5. The most widely used device in this category is the Graf ligament (Graf's ligament?), described by Henry Graf. ${ }^{[9]}$ The system consists of a non-elastic braided polyester ligament (dacrilene) in the form of a loop, which is applied round the pedicle screws (Figure 8). The ligaments are applied to the pedicle screws under tension to lock the motion segment in extension. The concept was that, once the facet joints were locked, it would stop rotation. The inventor Henri Graf was of the view that 'instability' was related to the development of an abnormal rotatory movement, and if this was stopped, the system would still allow limited flexion, but within the range of normal flexion, which would therefore probably not be painful. There was no clinical or

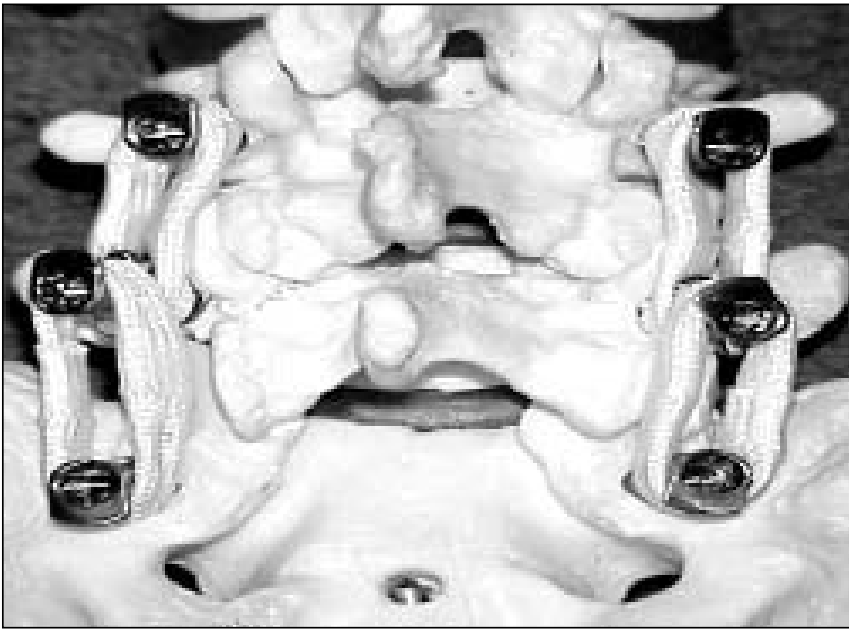

Figure 8: The Graf ligament system, applied between pedicle screws at L4-5 and L5-S1 segment in saw-bone ${ }^{[21]}$

experimental basis for this view as to the cause of instability, or the efficacy of the ligament. Despite this fact, Graf ligament has been used by several independent surgeons in Europe and far East, with clinical success comparable to that of fusion. ${ }^{[21,54-59]}$ The Graf's ligamentoplasty procedure also produces a significant increase in lateral canal stenosis, especially if there is any preexisting degenerative change in the facet joints or in-folding of the ligamentum flavum, owing to the marked lordosis of the segment instrumented, and indeed early clinical failure was often associated with this surgical complication. ${ }^{[48]}$ Graf ligament transfers the load from anterior aspect of the disc to the posterior annulus, and indeed increases the disc pressure profile towards the posterior annulus, which was further substantiated by experimental study with disc profilometry. ${ }^{[48]}$ It is somewhat uncertain as to whether the clinical success was related to the restriction of motion, or to the transfer of load to the posterior aspect removing loading from the painful anterior part of the disc. However, this may explain the late failure of Graf ligament, which accelerates the disc degeneration by overloading the posterior part of the disc. ${ }^{[21]}$

The dynesys (the dynamic neutralization system $)^{[60]}$ was developed by Gilles Dubois in 1994. In the present form, the system consists of titanium alloy (protasul 100) pedicle screws, polyester (sulene-PET) cords, and polycarbonaturethane (sulenePCU) spacers (Figure 9). The screws anchor the dynesys system in the pedicle and in the vertebral body. The modular spacer fits between the pedicle screw heads. The stabilizing cords connect the pedicle screw heads via the hollow core of the spacer in place. According to the authors, its preload provides uniform system rigidity. The stabilizing cord carries tensile forces and the spacers resist compressive forces. The system is said to establish a mobile load transfer and to control motion of the segment in all of orientation.

The biomechanical testing of the dynesys system, as published in the literature, is limited to fatigue testing of the whole construct, and biocompatibility of the non-metallic components. There is no data available for the load-sharing characteristics or kinematics 


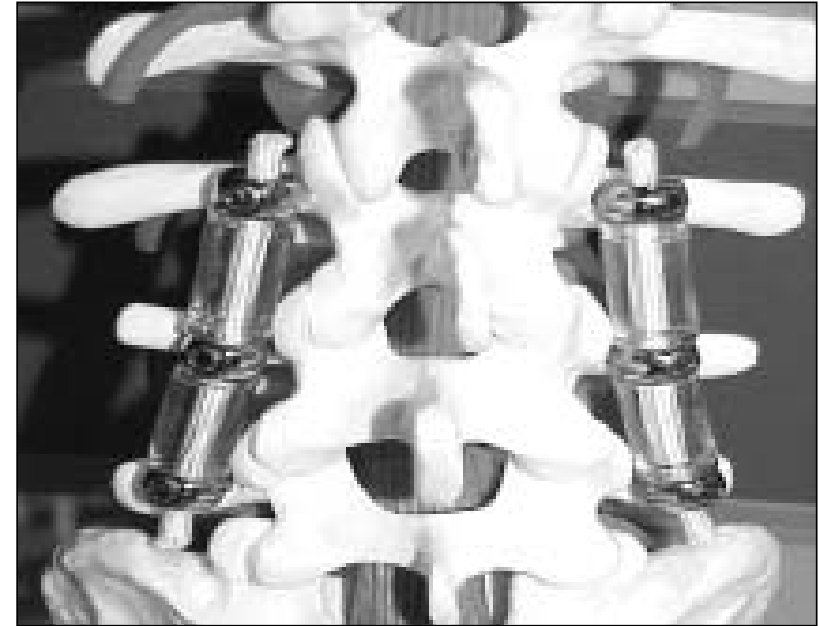

Figure 9: The dynesys system ${ }^{[21]}$

of the spinal motion segments after implantation of the dynesys system.

The authors presented their initial results on 83 patients. The indications included a variety of degenerative conditions including spinal stenosis, degenerative discopathy, disc herniation, spondylolisthesis, and revision surgery. The clinical results have been described to be encouraging and safe. However, unlike the Graf ligament, screw loosening was observed in seven cases. Early surgical intervention was needed in four cases, and late surgery was needed in five cases in the same segment and in seven cases for adjacent segment disease. The authors concluded that the dynamic neutralization proved to be a safe and effective alternative in the treatment of unstable lumbar conditions.

A closer look into the dynesys system clarifies that introduction of the spacers between the pedicle screws leads to a loss of the lordosis segment's. If the spinal extensor muscles can restore the lordosis of the segment, it will do so by distracting the disc space while the spacer acts as a load-bearing fulcrum. This would unload the disc. Rajaratnam et al. ${ }^{[61]}$ recently reviewed a group of 60 patients treated with dynesys, with postoperative standing films with a plumb line. They found that the patients who failed to benefit from the dynesys system are those who could not achieve lordosis at the stabilized segments. ${ }^{[61]}$ Therefore, the lordosis and load sharing by the plastic cylinder depends very critically on the placement of the implant, and on the ability of the patient to achieve lordosis with his extensor muscles. ${ }^{[21]}$

The FASS (fulcrum-assisted soft stabilization) system (Figure 10) was developed by the author ${ }^{[62]}$ to address what was perceived as disadvantages of the Graf system. There are two problems commonly experienced with the Graf system:

1. The lordosis that the Graf ligament system invariably produces results in narrowing of the lateral recess, leading to root entrapment, especially if there was any preexisting facet arthropathy.

2. The Graf system increases the loading of the posterior annulus, which is a feature of the painful degenerated disc.

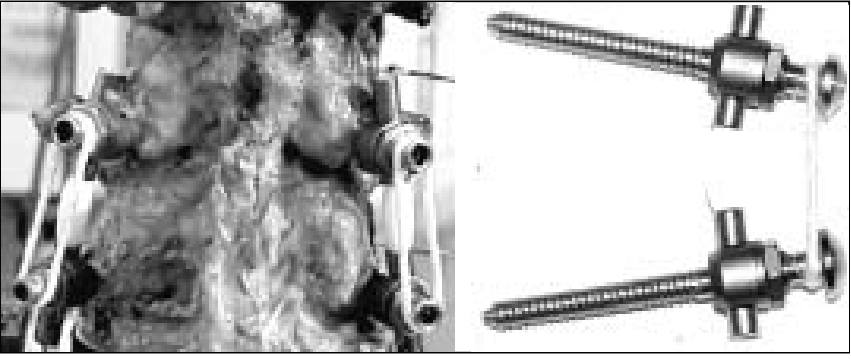

Figure 10: The FASS system, as applied to a cadaveric spine. In this prototype, the fulcrum is made up with flexible polytetrafluroethylene and the ligament is made up of elastic fabric band containing polyurethane

The presence of the fulcrum in the FASS system may prevent both these problems. The fulcrum is placed between the pedicle screws, in front of the ligament. The fulcrum distracts the posterior annulus. The elastic ligament is placed posterior to the fulcrum, to compress between the heads of the pedicle screws. The fulcrum transforms this posterior compression force into an anterior distraction force, which distracts and unloads the disc. It is imperative that the FASS device should be inserted with sufficient tension in the ligament to create the required lordosis.

The FASS system can unload the disc unaided by the posture or muscle action. It addresses a major disadvantage of the posterior interspinous distraction systems, insofar as it does not allow a segment to be put into kyphosis. The lordosis is created by the implant and is not dependent on the patient's ability to achieve lordosis with his or her own muscles.

The implant does experimentally unload the disc by sharing load. ${ }^{[62]}$ The degree of disc unloading depends on the relative tension and compression produced by the fulcrum and the ligament. For a given distraction by the fulcrum, the higher the compressive force applied by the ligament the greater would be the disc unloading. Laboratory experiments on spine models and cadaver spines demonstrate that greater disc unloading also develops higher stiffness of the motion segment. ${ }^{[63]}$ In other words, as greater unloading of the disc is achieved by adjustment of the tension in the ligament and the fulcrum, the system shares higher load, and the motion segment looses flexibility. Clearly, if a very stiff system is implanted, then screw loosening and implant failure is more likely to occur in the long term. To maintain the normal physiological environment in the disc, it may be desirable to unload the disc partially, and allow the segment mobility as close to normal as possible. The fulcrum should act as a load-sharing device and not a load-bearing device. The system is still under development (Sponsor: AO International, Davos, Switzerland) and a clinically applicable prototype is yet to be available.

6. Semi rigid metallic devices across the pedicle screws: currently there is no available soft stabilization system for clinical use, although there are a few under development.

The dynamic soft stabilization (DSS) system has been developed by the present author ${ }^{[47]}$ (Sponsor: Spinal Concepts Inc., Austin, TX), but has not yet been used clinically. Two designs of the DSS system have been tested in the laboratory. The DSS- 
I system consists of a titanium spring, made of spring grade titanium wires of $3 \mathrm{~mm}$ cross sectional diameter (Figure 11). The DSS-II system (Figure 11) consists of an elliptical coil spring, made from $4 \mathrm{~mm}$ spring grade titanium rods. Both the springs are applied to the motion segment by pedicle screws. A pretension at the spring during its application would share the load and unload the disc at rest. The flexibility of the spring will limit the flexibility of the motion segment. The disc unloading during motion will be dependent on the location of the optimum instant axis of rotation (IAR) of the springs and that of the motion segment. If the location of these IAR remains close to each other, the disc unloading and load sharing would be uniform throughout the range of motion, and will only depend on the flexibility of the spring. On the other hand, if there is a discrepancy in the relative location of the IAR, the DSS system may become an increasingly load-bearing structure at certain ranges of motion, which may lead to an early implant failure or loosening. Recent cadaver studies show that the IAR of the DSS-II system lies closer to that of the motion segment. Therefore it offers more favourable kinematics and promises to achieve the objectives of soft stabilization with optimum load sharing, disc unloading and control of motion.

\section{Soft stabilization as an adjunct to disc prosthesis}

As stated earlier, disc replacement is only an equivalent to a partial joint replacement. In the presence of significant facet joint arthritis, disc replacement may not relieve pain. When radicular pain warrants decompression involving partial facetectomy, a disc replacement may destabilize the motion segment. A posterior soft stabilization system promises to add stability after decompressive lumbar laminectomy or facetectomy. In effect, addition of a posterior soft stabilization to disc replacement may make it equivalent to total joint replacement.

\section{Soft stabilization and disc repair}

If a favourably environment may be created in the motion

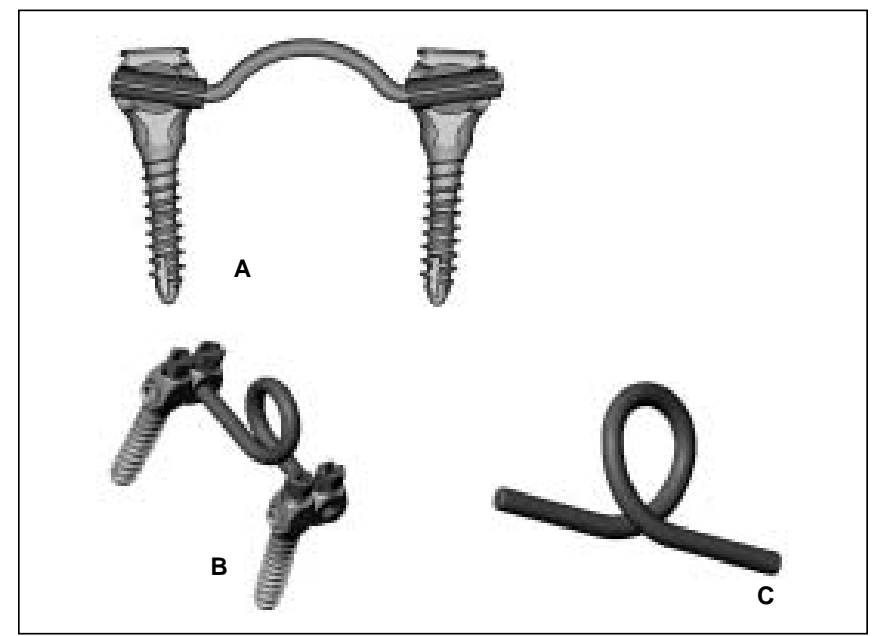

Figure 11: DSS-I (A) system consists of titanium spring in the form of a 'C', and DSS-II system (B) consists of a titanium coil spring, which can be attached to the pedicle screws of different designs segment by unloading the disc and permitting near normal motion, the disc may be able to repair itself. Gene therapy in degenerative disc diseases, either by promoting enzymes to produce proteoglycans, or by preventing the enzymes like proteases, which damage the disc, is an emerging technology with a lot of promise. Soft stabilization may further enhance the reparative process activated by the gene therapy.

\section{Conclusion}

While soft stabilization appears to be promising, we should take a cautious approach to any new implant system. This implant for fusion only has to serve a temporary stabilization until fusion has taken place; on the other hand, a soft stabilization system has to provide stability throughout its life. Implant loosening is common in the presence of pseudarthrodesis. After soft stabilization, the implant has to stay anchored to the bone despite allowing movement. This sounds like an incredible task. However, the flexibility of the implant system should be able to protect it from loosening at the anchor point into the bone. Finally, the soft stabilization system is intended to load share with the disc and the facet joint only partially and unloads the motion segment. However, any mismatch between the kinematics of the implant system and the motion segment, in particular discrepancy between their IAR, would result in the implant bearing unexpected load at certain ranges of motion. If that happens, it would guarantee an early implant failure or loosening. The need for a strict bench test in the laboratory, therefore, cannot be overemphasized. The few soft stabilization systems that have had clinical applications so far produced a clinical outcome comparable to that of fusion. No prospective randomised controlled trial has been reported yet, which is an essential need for practice of evidence-based medicine.

\section{References}

1. Boos N, Webb JK. Pedicle screw fixation in spinal disorders: a European view. Eur Spine J 1997;6:2-18.

2. Gibson JN, Grant IC, Waddell G. The Cochrane review of surgery for lumbar disc prolapse and degenerative lumbar spondylosis. Spine 1999;24:1820-32.

3. Chou WY, Hsu CJ, Chang WN, Wong CY. Adjacent segment degeneration after lumbar spinal posterolateral fusion with instrumentation in elderly patients. Arch Orthop Trauma Surg 2002;122:39-43.

4. Eck JC, Humphreys SC, Hodges SD. Adjacent-segment degeneration after lumbar fusion: a review of clinical, biomechanical, and radiologic studies. Am J Orthop 1999;28:336-40.

5. Etebar S, Cahill DW. Risk factors for adjacent-segment failure following lumbar fixation with rigid instrumentation for degenerative instability. J Neurosurg Spine 1999;90:163-9.

6. Kumar MN, Baklanov A, Chopin D. Correlation between sagittal plane changes and adjacent segment degeneration following lumbar spine fusion. Eur Spine $J$ 2001;10:314-9.

7. Markwalder TM, Wenger M. Adjacent-segment morbidity. J Neurosurg Spine 2002;96:139-40.

8. Rahm MD, Hall BB. Adjacent-segment degeneration after lumbar fusion with instrumentation: a retrospective study. J Spinal Disord 1996;9:392-400.

9. Graf H. Lumbar instability. Surgical treatment without fusion. Rachis 1992;412:123-37.

10. Mochida J, Suzuki K, Chiba M. How to stabilize a single level lesion of degenerative lumbar spondylolisthesis. Clin Orthop 1999;(368):126-34

11. Fujiwara A, Lim TH, An HS, Tanaka N, Jeon CH, Andersson GB, et al. The effect of disc degeneration and facet joint osteoarthritis on the segmental flexibility of the 
lumbar spine. Spine 2000;25:3036-44.

12. Fujiwara A, Tamai K, An HS, Kurihashi T, Lim TH, Yoshida H, et al. The relationship between disc degeneration, facet joint osteoarthritis, and stability of the degenerative lumbar spine. J Spinal Disord 2000;13:444-50.

13. Friberg O. Lumbar instability: a dynamic approach by traction-compression radiography. Spine 1987;12:119-29.

14. Ogston NG, King G.J, Gertzbein SD, Tile M, Kapasouri A, Rubenstein JD. Centrode patterns in the lumbar spine. Baseline studies in normal subjects. Spine $1986 ; 11: 591-5$

15. Okawa A, Shinomiva K, Komori H, Muneta T, Arai Y, Nakai O. Dynamic motion study of the whole lumbar spine by videofluoroscopy. Spine $1998 ; 23: 1743-9$.

16. Seligman JV, Gertzbein SD, Tile M, Kapasouri A. Computer analysis of spinal segment motion in degenerative dise disease with and without axial loading. Spine $1984 ; 9: 566-73$

17. Stokes IA, Frymoyer JW. Segmental motion and instability. Spine 1987;12:68891.

18. Weiler PJ, King GJ, Gertzbein SD. Analysis of sagittal plane instability of the lumbar spine in vivo. Spine 1990;15:1300-6.

19. Panjabi M, Yamamoto I, Oxland T, Crisco J. How does posture affect coupling in the lumbar spine? Spine 1989;14:1002-11.

20. Pearcy M, Portek I, Shepherd J. The effect of low-back pain on lumbar spinal movements measured by three-dimensional X-ray analysis. Spine 1985;10:1503.

21. Mulholland RC, Sengupta DK. Rationale, principles and experimental evaluation of the concept of soft stabilization. Eur Spine J 2002;11 Suppl 2:S198-205.

22. Nachemson AL. Disc pressure measurements. Spine 1981;6:93-7.

23. Wilke H, Neef P, Hinz B, Seidel H, Claes L. Intradiscal pressure together with anthropometric data- a data set for the validation of models. Clin Biomech (Bristol, Avon) 2001;16 Suppl 1:S111-26.

24. Hukins DW. A simple model for the function of proteoglycans and collagen in the response to compression of the intervertebral disc. Proc R Soc Lond B Biol Sci 1992;249:281-5.

25. MeMillan DW, MeNally DS, Garbutt G, Adams MA. Stress distributions inside intervertebral dises: the validity of experimental stress profilometry. Proc Inst Mech Eng [H] 1996;210:81-7.

26. MeNally DS, Adams MA. Internal intervertebral disc mechanies as revealed by stress profilometry. Spine 1992;17:66-73.

27. MeNally DS, Shackleford IM, Goodship AE, Mulholland RC. In vivo stress measurement can predict pain on discography. Spine 1996;21:2580-7.

28. Krag MH, Seroussi RE, Wilder DG, Pope MH. Internal displacement distribution from in vitro loading of human thoracic and lumbar spinal motion segments experimental results and theoretical predictions. Spine 1987;12:1001-7.

29. Moore RJ, Vernon-Roberts B, Fraser RD, Osti OL, Schembri M. The origin and fate of herniated lumbar intervertebral disc tissue. Spine 1996;21:2149-55.

30. Ariga K, Miyamoto S, Nakase T, Okuda S, Meng W, Yonenobu K, et al. The relationship between apoptosis of endplate chondrocytes and aging and degeneration of the intervertebral disc. Spine 2001;26:2414-20.

31. Keller TS, Hansson TH, Abram AC, Spengler DM, Panjabi MM. Regional variations in the compressive properties of lumbar vertebral trabeculae. Effects of disc degeneration. Spine 1989;14:1012-9

32. Simpson EK, Parkinson IH, Manthey B, Fazzalari NL. Intervertebral disc disorganization is related to trabecular bone architecture in the lumbar spine. J Bone Miner Res 2001;16:681-7.

33. MeNally DS. The objectives for the mechanical evaluation of spinal instrumentation have changed. Eur Spine J 2002;11 Suppl 2:S179-85.

34. Fritzell P, Hagg O, Wessberg P, Nordwall A. Chronic low back pain and fusion: a comparison of three surgical techniques: a prospective multicenter randomized study from the Swedish lumbar spine study group. Spine 2002;27:1131-41.

35. Agazzi S, Reverdin A, May D. Posterior lumbar interbody fusion with cages: An independent review of 71 cases. .J Neurosurg Spine 1999;91:186-92.

36. Bjarke Christensen F, Stender Hansen E, Laursen M, Thomsen K, Bunger CE. Long-term functional outcome of pedicle screw instrumentation as a support for posterolateral spinal fusion: randomized clinical study with a 5-year follow-up. Spine $2002 ; 27: 1269-77$.

37. Christensen FB, Thomsen K, Eiskjaer SP, Gelinick J, Bunger CE. Functiona outcome after posterolateral spinal fusion using pedicle screws: Comparison between primary and salvage procedure. Eur Spine J 1998;7:321-7.

38. Dahl B, Gehrchen P, Blyme P, Kiaer T, Tondevold E. Clinical outcome after spinal fusion with a rigid versus a semi-rigid pedicle screw system. Eur Spine J $1997 ; 6: 412-6$.

39. Kawakami M, Tamaki T, Ando M, Yamada H, Hashizume H, Yoshida M. Lumbar sagittal balance influences the clinical outcome after decompression and posterolateral spinal fusion for degenerative lumbar spondylolisthesis. Spine $2002 ; 27: 59-64$

40. Korsgaard M, Christensen FB, Thomsen K, Hansen ES, Bunger C. The influence of lumbar lordosis on spinal fusion and functional outcome after posterolateral spinal fusion with and without pedicle screw instrumentation. J Spinal Disord Tech 2002;15:187-92

41. Mahvi DU, Zdeblick TA A prospective study of laparoseopic spinal fusion. Technique and operative complications. Ann Surg 1996;224:85-90.

42. Thomsen K, Christensen FB, Eiskjaer SP, Hansen ES, Fruensgaard S, Bunger CE. 1997 Volvo Award winner in clinical studies. The effect of pedicle screw instrumentation on functional outcome and fusion rates in posterolateral lumbar spinal fusion: a prospective, randomized clinical study. Spine 1997;22:281322 .

43. Whitecloud TS 3rd, Castro FP Jr, Brinker MR, Hartzog CW Jr, Ricciardi JE, Hill C. Degenerative conditions of the lumbar spine treated with intervertebral titanium cages and posterior instrumentation for circumferential fusion. J Spinal Disord 1998;11:479-86.

44. McAfee PC. Interbody fusion cages in reconstructive operations on the spine. .J Bone Joint Surg Am 1999;81:859-80.

45. Lazennec JY, et al. Sagittal alignment in lumbosacral fusion: relations between radiological parameters and pain. Eur Spine .J 2000;9:47-55.

46. Polikeit AOT, Nolte LP. Factors effecting the behaviour of Interbody cages in the lumbar spine - finite element analysis. ISSLS Annual Meeting; 2000 Apr 19-23; Adelaide, Australia.

47. Sengupta DK, Herkowitz HN, Hochschuler S, Mulholland RC. Loads sharing characteristics of two novel soft stabilization devices in the lumbar motion segments - a biomechanical study in cadaver spine. Spine Arthroplasty Society Annual Conference; 2003 May 5; Scottsdale, USA

48. Grevitt MP, Gardner AD, Spilsbury J, Shackleford IM, Baskerville R, Pursell LM, et al. The Graf stabilisation system: early results in 50 patients. Eur Spine $\mathrm{J}$ $1995 ; 4: 169-75$

49. Minns R.J, Walsh WK. Preliminary design and experimental studies of a novel soft implant for correcting sagittal plane instability in the lumbar spine. Spine $1997 ; 22: 1819-25$.

50. Senegas .J, Etchevers .JP, Vital JM, Baulny D, Grenier F. Recalibration of the lumbar canal, an alternative to laminectomy in the treatment of lumbar canal stenosis. Rev Chir Orthop Reparatrice Appar Mot 1988;74:15-22.

51. St. Francis Medical Technologies I. The X STOP interspinous process distraction system (IPD) for the treatment of lumbar spinal stenosis [cited 2001]; 2001. Available from: www.sfmt.com/ipd.html

52. Caserta S, La Maida GA, Misaggi B, Peroni D, Pietrabissa R, Raimondi MT, et al Elastic stabilization alone or combined with rigid fusion in spinal surgery: A biomechanical study and clinical experience based on 82 cases. Eur Spine .J 2002;11 Suppl 2:S192-7.

53. Garner MD, Wolfe SJ, Kuslich SD. Development and preclinical testing of a new tension-band device for the spine: The Loop system. Eur Spine J 2002;11 Suppl 2:S186-91.

54. Brechbuhler D, Markwalder TM, Braun M. Surgical results after soft system stabilization of the lumbar spine in degenerative disc disease-long-term results. Acta Neurochir (Wien) 1998;140:521-5.

55. Gardner A, Pande KC. Graf ligamentoplasty: A 7-year follow-up. Eur Spine J 2002;11 Suppl 2:S157-63.

56. Guigui P, Chopin D. Assessment of the use of the Graf ligamentoplasty in the surgical treatment of lumbar spinal stenosis. Apropos of a series of 26 patients. Rev Chir Orthop Reparatrice Appar Mot 1994;80:681-8.

57. Hadlow SV, Fagan AB, Hillier TM, Fraser RD. The Graf ligamentoplasty procedure. Comparison with posterolateral fusion in the management of low back pain. Spine 1998; 23:1172-9.

58. Kanayama M, Hashimoto T, Shigenobu K, Harada M, Oha F, Ohkoshi Y, et al. Adjacent-segment morbidity after Graf ligamentoplasty compared with posterolateral lumbar fusion. J Neurosurg Spine 2001;95:5-10.

59. Legaye J, De Cloedt P, Emery R. Supple intervertebral stabilization according to Graf. Evaluation of its use and technical approach. Acta Orthop Belg 1994;60:393401.

60. Stoll TM, Dubois G, Schwarzenbach $\mathrm{O}$. The dynamic neutralization system for the spine: a multi-center study of a novel non-fusion system. Eur Spine J 2002;11 Suppl 2:S170-8.

61. Rajaratnam SS, Mueller M, Shepperd JAN, Mulholland RC. Dynesis stabilization of the lumbo-sacral spine. Britspine 2002, The Second Combined Meeting of the BSS BASS BCSS SBPR; 2002 Feb 27-Mar 1; Birmingham, UK.

62. Sengupta DK, Guyer RD, Hochschuler S, Mulholland RC. Fulcrum assisted soft stabilisation in the in the treatment of low back pain - a new concept. ISSLS Annual Meeting; 1999 Jun 21-25; Hawaii, USA.

63. Sengupta DK, Webb JK, Mulholland RC. Can soft stabilization in the lumbar spine unload the disc and retain mobility? A biomechanical study with fulerum assisted soft stabilization on cadaver spine. ISSLS Annual Meeting; 2001 Jun 19-22; Edinburgh, Scotland. 